\title{
THE NUMERICAL ANALYSIS OF MARINE-SHIP PROPELLER
}

\author{
SIDDHARDHA NAIDU GORJA ${ }^{1}$, JAGADEESK KOTA ${ }^{2} \&$ SRINIVAS. $\mathbf{G}^{3 *}$ \\ ${ }^{1,2}$ Student, Department of Aeronautical and Automobile Engineering, \\ Manipal Institute of Technology, Manipal, Karnataka, India \\ ${ }^{3}$ Assistant Professor, Department of Aeronautical and Automobile Engineering, \\ Manipal Institute of Technology, Manipal, Karnataka, India
}

\begin{abstract}
In this paper, numerical simulationwas done to decide the hydrodynamic qualities in a cavitatingviscous stream of the standard INSEAN E779A in single and couple propeller arrangement utilizing cavitation show executed in FLUENT Software. Next, figuring's have been done on fore propeller of tandem propeller and tandem propeller in non-cavitating streams. Those registered exhibitions indicate great concurrence with trial information. Next, the numerical methodology was connected in loaded conditions to the instance of couple propeller arrangements individually 0.6 and 0.2 pivotal uprooting. The outcomes uncover the comparison of thrust and torque between the single propeller and tandem propeller. The utilization of pair co-turning propeller in stacked conditions is featured.
\end{abstract}

KEYWORDS: Marine Propeller, Cavitation \& Tandem Propeller

Received: May 18, 2019; Accepted: Jun 08, 2019; Published: Jul 18, 2019; Paper Id.: IJMPERDAUG2019113

\section{INTRODUCTION}

A propeller is a rotating device with multiple which uses the power generated and transferred by the primary motor of the marine-vehicle. This power is converted to revolutionary motion to create a force which gives energy to the water and generates power which helps the ship in moving forward.

Cavitation impact sly affects marine propellers. Without a doubt, it causes the disintegration of the cutting edge in this manner devastates the propeller. Besides, it can influence the effectiveness and the push, extensively constraining the activity of impetus framework. The expenses of the frameworks engaged with the ship impetus that may be harmed by this marvel are colossal. It is hard to keep away from totally these impacts however decreasing it however much as could reasonably be expected. To do as such, more consideration ought to be given for the plan period of the propeller.

Efficiency of the propeller is calculated from the formula.

$$
\eta_{0}=\frac{T o U}{2 \pi n_{p} Q_{p}}
$$

\section{LITERATURE REVIEW}

In this paper, D. Boucetta et al [1] a numerical recreation was made to choose the hydrodynamic qualities in the cavitation of the thick progression of the INSEAN E779A standard helix in a couple and partner strategy using Singhal et al. The cavitation show executed in ANSYS. From that point forward, calculations have been made on a single propeller in currents with and without cavitation. The shows inscribed have exhibited an extraordinary 
synchronization with fundamental information. Next, the numerical theory has been related in stacked conditions to the example of a pair of planes of helices with an essential ejection of 0.6 and 0.2 . The results reveal that cavitation is estimated dynamically, this cavitation zone was articulated sensibly in the front propeller. The use of torque co-torque propeller in stacked conditions is incorporated.

Anna Maria Kozlowska et al [2], introduced an exchange of the figure of air starting and air extraction of the propellers of the ships, planning to imagine beneath conditions the ventilation and the authentic physical piece of the ventilation will take place. Three notable forms of ventilation source structures are intertwined in our arguments: free-surface vortex ventilation, ventilation by aspirating without the free surface enclosing a vortex as well as venting with propeller emerging out of water. The figure of ventilation depended on the development of model tests, where the helix was tried on several components of sporadic ventilation. Utilization of submerged video tape gives a perceptible understanding of ventilation that is considered. The vortex advance ventilation has analogies with several supernatural occurrences, for example, the straight vortex in the siphon sumps, the ground vortex at the entrance of carrier motors and the Vortex cavitation of helix of the propeller (PHVC). The association of paper cables between the free surface vortex ventilation (PFSVV) of PHVC and the propeller-free surface comparatively as an examination between the PFSVV courses of action and the vortex of the air motors in between of a high-power task is a strong surface. The exploratory information subject to two or three tests of specific models shows the cut between the vortex conformation, the inclusion of no vortex and free surface ventilation flow plans. For the association, the particular variables are used, which selected the strength of the hydrodynamic joint among the helix and the free surface.

The aim of Qin Wu et al [3] was to numerically inspect the cavitation current around a marine helix and investigate the innate associations between the cavitation of the foil and its noise due to radiation. The irregularity of the K- $\omega$ SST exhibits an unpleasant effect on the thickness cure and Zwart's demonstration of cavitation are familiar with the recreation of the cavitation current throughout a helix in a non-uniform wake. Stacking clutter and noise due to cavitation are estimated based on this theory of the acoustic fan source and the speculation of sound radiation for round air storage independently. The irregular advancement of cavitation has been achieved, and the gigantic incidental weight instability around the sharp edge has been penniless with the general frequencies in understanding the most important request of the first repeat line of passage. In the case of non-cavitation, the high and strong weight levels generally accumulate in the low-lying repetition organization and reduction from the low request to the repetition of the acute edge step of high demand. Whereas, for the case of sheet cavitation, the noise weight levels at the high request for edge step frequencies is improved.

The choice of a perfect marine propeller was a champion among the most basic themes of the oceanic plan. This problem can be solved using the helix plan designs or the backward polynomials. M.M. Gaafary et al [4] know a system to find the perfect properties of the marine helices of B-course of action. The process of disposing the propeller was executed as a single-purpose work presented to constraints restricted by cavitation, the quality of the material and the required thrust of the propeller. Regardless of how we can capture the improvement of business programming to address the problem, the PC program that has been made in a remarkable way for this effort can be progressively important because of its elasticity and credibility to join, as a subroutine, with the incredible process of marine-ship layout.

Different kinds of cavitation showing up on the outside of a propeller when the marine-ship progresses with a rapid reason the execution of propeller's decreases as well as harms (disintegrations) to the propeller. Along these lines, 
better knowledge of the cavitation arrangements and the capacity to foresee them are vital for the structure of an effective propeller. Yu-chi Chang et al [5] presents picture consequences of cavitation acquired on the suction surfaces of a particular propeller and breaks down the pictures to get the factual variables of cavitations, for example, areas, sizes, and their involved territories.

The submerged commotion has generated a colossal proportion of enthusiasm during the last decade due to its potential impact on marine fauna. The business dispatch was one of the critical suppliers. Among several prepared sources, cavitation directions, the general levels of agitation radiated beyond its onset. Apart from its centrality, the instruments that drive the noise and repetition zones related to cavitation in which the particular components of cavitation contribute are not sufficiently considered. To address this openness in the composition, Batuhanaktas et al [6] coordinated an examination to give strategies for a possible methodology of desire of cavitation of the propeller and to improve the understanding of the cavitation frameworks of the disturbance by applying a proper sign. of procedures. Within this framework, this examination presents a repeated time-lapse examination procedure driven to contemplate a tumult driven by cavitation through the use of standard game plan helix data. The productive cavitation tunnel test is performed with six people from the course of action of the Meridian Standard propeller that are intentionally selected to consider the effect of the critical variables of the propeller plan, for example, the ratio of the area of the blade (BAR), the degree of inclination to the diameter (P/D) and the sharp edge number.

Different marine-ship propellers were secured with an unfriendly fouling paint, to be an explicit failure release, to improve the profitability of the propeller due to the enthusiastic increases in fuel costs. This late start has been joined by tougher natural rules that drive the transportation system to check its adversary for fouling techniques. With the introduction of anticoatings from another era, it was now feasible to spread the coatings not only to keep the edge free of biofouling, but also on the propellers to upgrade its efficiency. Within the above configuration, an examination was completed on the effect of the coatings on the hydrodynamic spectacles of the marine propellers. This document covers the impact of soil reveal coatings on the viability, cavitation and noise characteristics of a model-scale marine-ship propeller. EminKorkut et al [7] joined the nuances of these coordinated tests in the Emerson Cavitation Tunnel of the University of Newcastle and the discussions on the application of the paint, the problems of surface representation and the examination of the test results to determine safe and uncoated conditions. The outcomes showed the coverage cannot effect any decline in execution when properly associated.

The distinctive varieties of cavitation that appear on the outside of a propeller when the marine-ship proceed due to the rapid reason of the root of the propeller's execution or possibly damage (deterioration) of the propeller. In this sense, a better understanding of cavitation plans and the ability to predict them are the basics to a convincing helix structure. Yu-chi Chang et al [8] showed delayed images of the consequences of cavitation achieved on the suction surfaces of a particular helix and separated those photos to ensure the actual variables of the cavitation, for example, their territories, sizes and zones included.

YiannisVentikos et al [9] presented a numerical method to estimate the cavitation currents around the bodies of elevation. The computation uses a Navier Stokes enthalpy solver from a fluid which can manage the different parameters of the fluid, close to the state laws of water vapor mixing conveniently arranged, to talk about the flow of water and steam of two organized and the change that begins with a phase. In the next. The strategy reflects on the deviation of suffering and the uncertain, 2-D and cavitating currents for less and average Reynolds numbers. The work of the strategy for several 
analyzes demonstrates an extraordinary simultaneity with the estimates and insights of the open tests and shows captivating wonders related to cavitation and its correspondence with sticky instruments.

In practical conditions of the ocean, the shipping schemes undergo a great development due to the wave actions, which can also cause the propellers of the ship to influence the incorporation of water. To examine the development of a marine-ship propeller that works at the rear of a surface vessel that crosses among waves, Lianzhou Wang et al [10] coordinated a numerical entertainment in a helix influenced by the development of the launch in a current of cavitation utilizing the Reynolds. Methodology of Navier-Stokes (RANS). The rotation of the helix and the translation was carried out utilizing the stages of development described in this way. The development of the impulse was improved as a discontinuous development subject to a sinusoidal limit. The numerical information is transferred from the non-reliable flow field was practiced using the oversized system approach. The unsafe impulse coefficient and the torque coefficient of the helices are examined at different times of thrust development. A detailed report was made on the unsafe execution of cavitation and the activation features of the helix. With the development of the helix of the propeller, the field of the current is not coherent and constantly changes the storage in the helix in the middle of each period of change and each launch period, the load of the propeller and the field of wake are solidly related to the variety of the development period of the propeller.

\section{SUMMARY}

The majority of the papers which include in the trial investigation on ship propellers manage the threat of free surface ventilation and vortex ventilation of the propellers. It was demonstrated, that the vortex shaping instrument of Propeller Hull Vortex Cavitation (PHVC) is firmly identified with the system of Propeller Free Surface Vortex Ventillation (PFSV). The event of the PHVC and PFSVV relies upon the load coefficient CT, tip leeway proportion C/D and stream cavitation or ventilation number. In the vast majority of the cases the creators would run CFD examination on the test models of the propellers and gain the outcomes and contrast them and real viable propellers which are right now under use, along these lines improving the execution of the propellers by supplanting with the compelling information got from the test investigation. In the numerical examination the greater part of the creators attempted to inspect the issues like diminishing of proficiency amid rapid applications, stacking commotion, cavitation clamor, vortex wake, and different issues identified with propeller component by calculating the numerical models by a limited volume code. At long last, the aftereffects of recreation the propeller's wake example and 3D stream around the propeller, with and without bank are thought about. The impact of these parameter changings in the working propeller execution is painstakingly considered and broke down.

\section{METHODOLOGY}

\section{Geometry}

A flow of 3-dimentional flow has been made showing the use of CFD to inspect the presence of cavitation flow as a limit in working conditions. Two propeller devices have been considered that depend on the INSEAN E779A standard, a single and tandem propeller configuration. The essential geometrical parameters of the helices suggestions are abbreviated below. The helices used in the geometries of the pair have idem widths and the respective vital migration among the rear and the front propeller was proportional to 0.2 and 0.6 . The ejection of the betting partner between the propellers remained 0 degree. Figure-1 depicts the geometrical condition of the propeller in simple and torque designs made with Gambit. 
Table 1

\begin{tabular}{|l|c|c|}
\hline \multicolumn{1}{|c|}{ Model Name } & $\begin{array}{c}\text { INSEAN E779A } \\
\text { Single Propeller }\end{array}$ & $\begin{array}{c}\text { Tandem co-Rotating } \\
\text { Propeller }\end{array}$ \\
\hline Blades number & 4 & $(4+4)$ \\
\hline Diameter $(\mathrm{m})$ & 0.22727 & 0.22727 \\
\hline Pitch ratio at 0.7R & 1.1 & $($ PFore/D)=(PAft/D)- 0.2 \\
\hline Expanded area ratio & 0.689 & $(0.689+0.689)$ \\
\hline Skew & $4^{\circ} 48^{\prime}$ & $4^{\circ} 48^{\prime}$ \\
\hline Rake & $4^{\circ} 35^{\prime}$ & $4^{\circ} 35^{\prime}$ \\
\hline
\end{tabular}

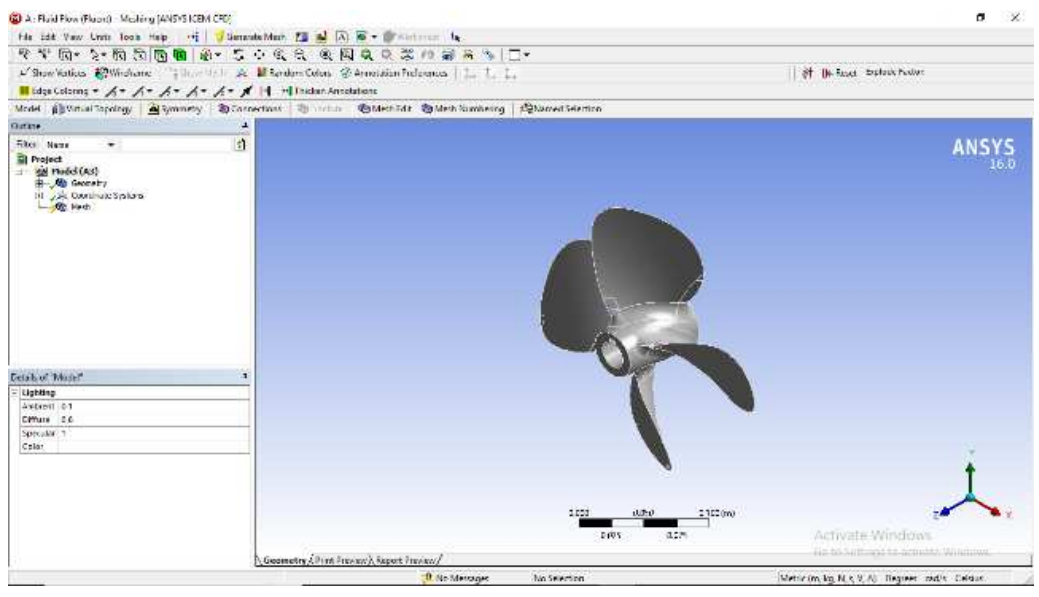

Figure 1: Fore Propeller of the Tandem Propeller (E779A)

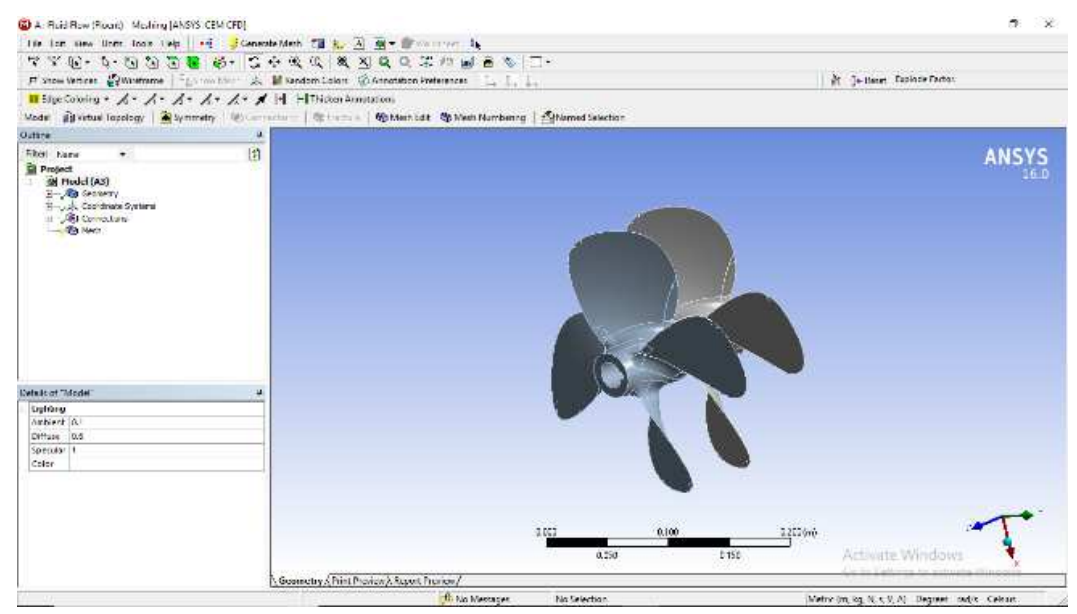

Figure 1: Tandem Propeller (E779A)

\section{Meshing}

Taking into account the condition of periodicity, the computation area was made for a single blade. Due to the multifaceted nature of the design of the propellers of marine vessels, unstructured tetrahedral work was received utilizing the Gambit preprocessor. The work area was characterized at the blade of a propeller. The work occurred so that the cell sizes near the sharp edge divider were small and logically expanded to the outer limit. Finally, the estimation area adjusted by tetrahedral networks has been acquired. Due to cavitation, the work is organized so that it is not too thin (limit of layers) to avoid too unnecessary counting times and matches the display of selected disturbances (Standard K-). In addition, specific consideration was given to controlling the asymmetry of the components to detect a decent nature of the work created. The number of cells along these produced lines is 903,000 proportional to several 182,000 hubs. 


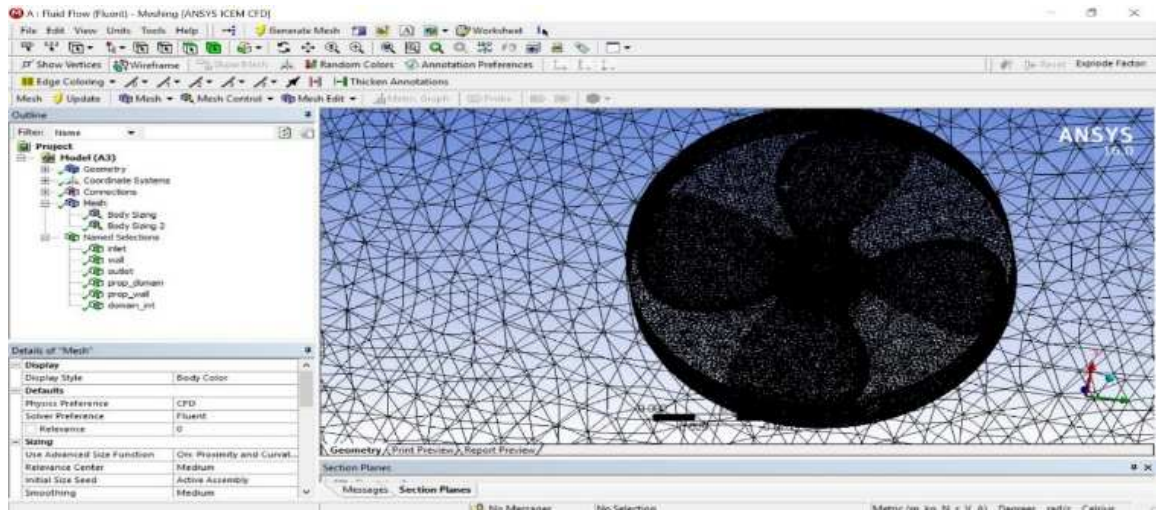

Figure 2: Meshing (1)

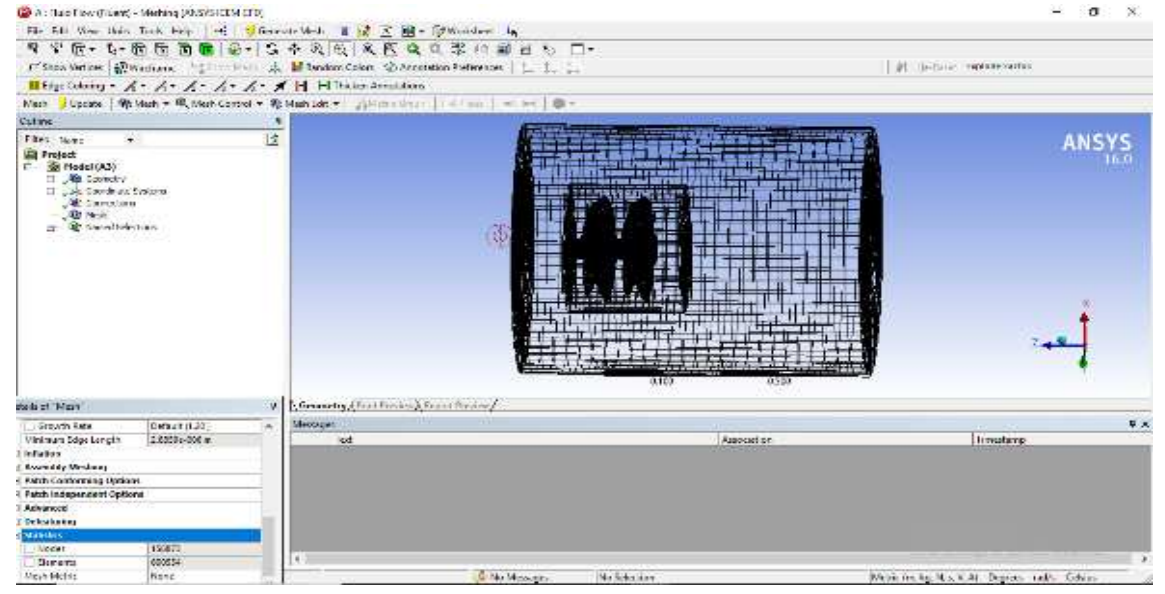

Figure 3: Meshing (2)

\section{Boundary Conditions}

Table 2

\begin{tabular}{|l|l|}
\hline Velocity inlet & $1 \mathrm{~m} / \mathrm{s}$ \\
\hline Pressure outlet & 0 gauge \\
\hline Operating pressure & $1 \mathrm{~atm}$ \\
\hline Turbulence model & Standard k-e, standard wall function \\
\hline multiphase & Eulerian, mass transfer mechanism - cavitation \\
\hline Frame motion & $36 \mathrm{rps}$ \\
\hline Vessel speed & $0.9 \mathrm{~m} / \mathrm{s}$ \\
\hline Propeller rotation & $36 \mathrm{rps}$ \\
\hline
\end{tabular}

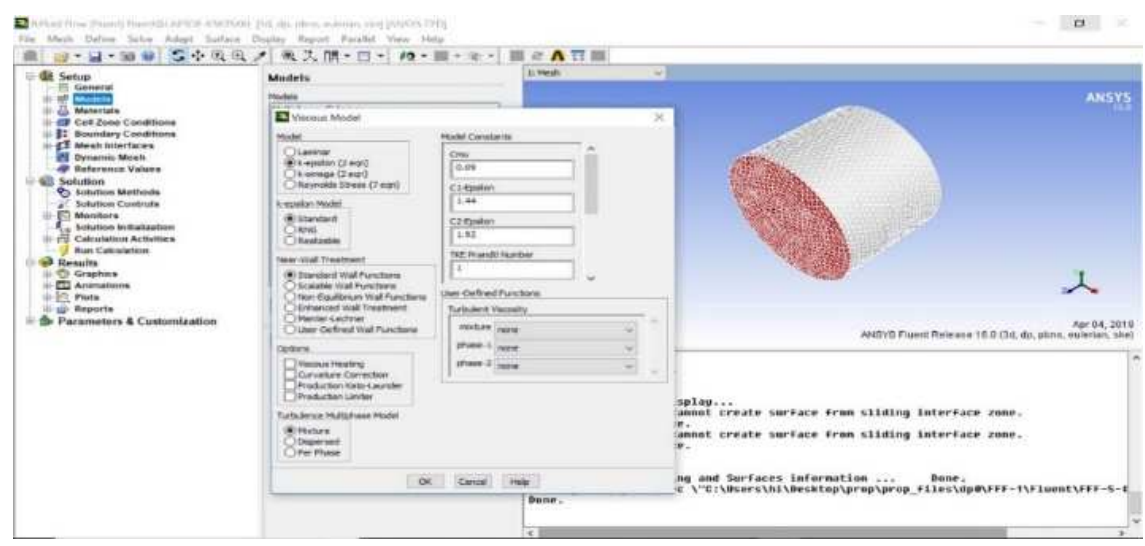

Figure 4: Boundary Condition (1) 


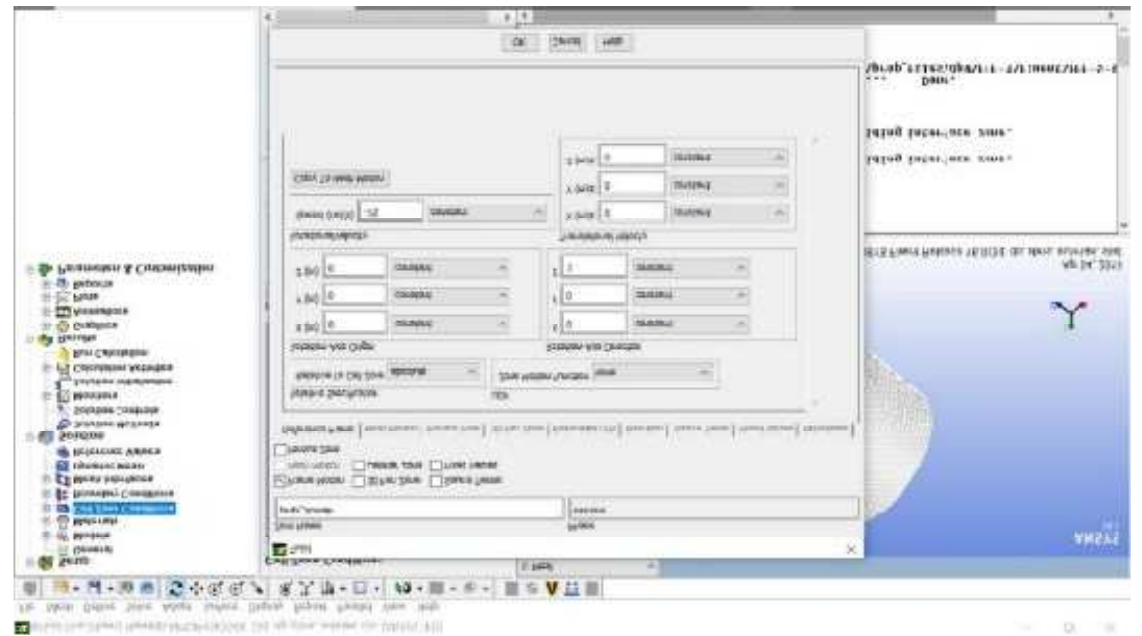

Figure 5: Boundary condition (2)

\section{RESULTS AND DISSCUSSIONS}

Force and Torque Solution was run with suitable iterations as shown in [Figure 7 - 10]. These Results are well suited for validation of numerical codes.

The values of force (thrust) and torque were obtained in all the directions i.e. X, Y, Z and the coefficients of thrust, torque and efficiency of the fore propeller of the tandem propeller are calculated and compare with values obtained in the reference paper.

$$
\eta_{0}=\frac{T o U}{2 \pi n_{p} Q_{p}} K_{T}=\frac{T_{0}}{\rho n_{p}^{2} D^{4}} K_{Q}=\frac{Q_{p 0}}{\rho n_{p}^{2} D^{5}}
$$

The above formulae are used to obtain the coefficients and efficiency.

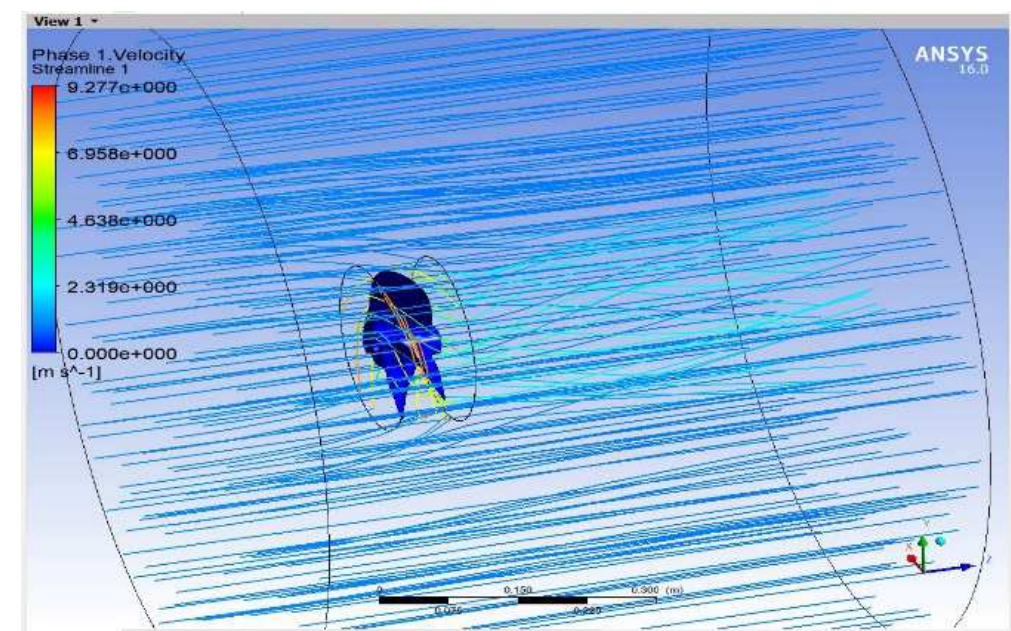

Figure 6: Velocity of a Single Propeller 


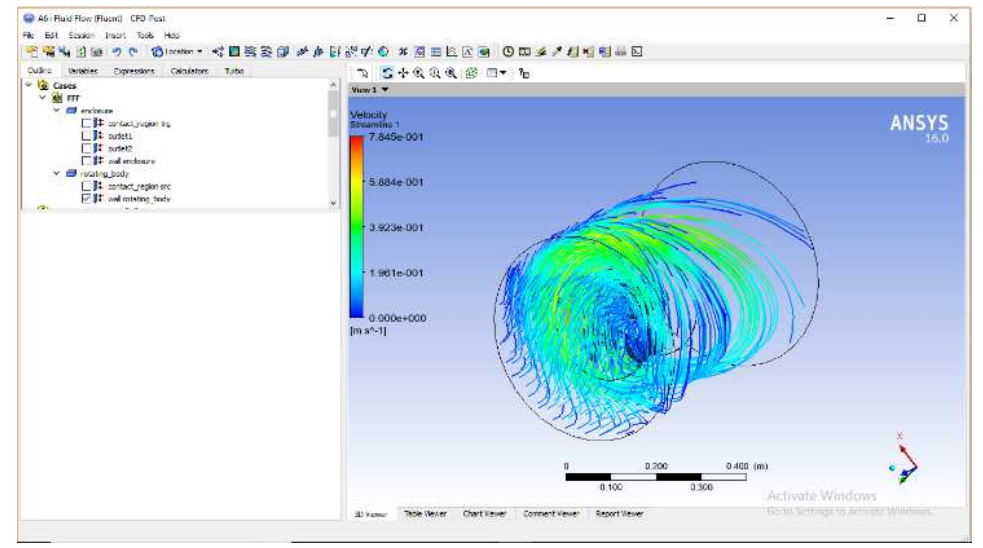

Figure 7: Velocity of the Tandem Propeller

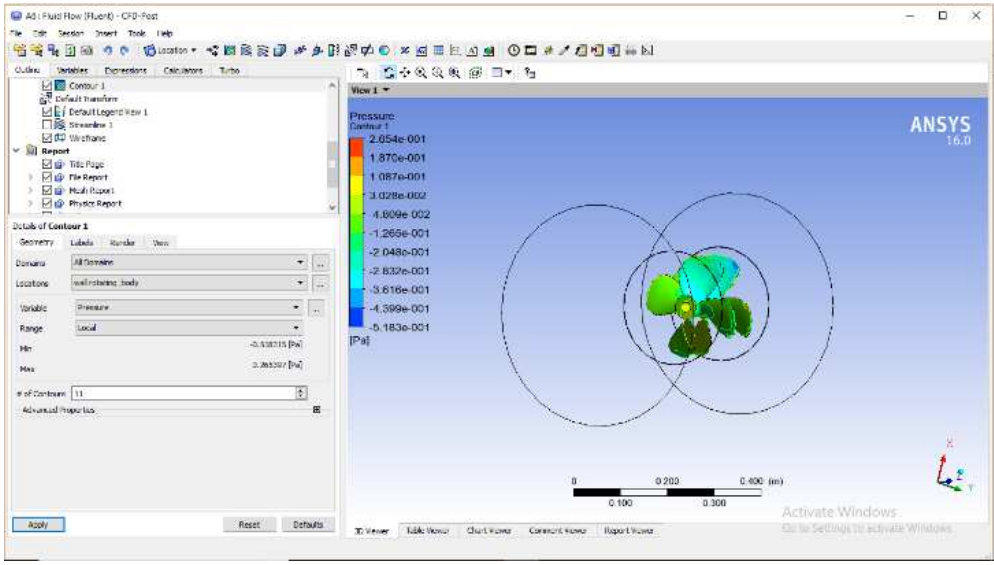

Figure 8: Pressure of Tandem Propeller

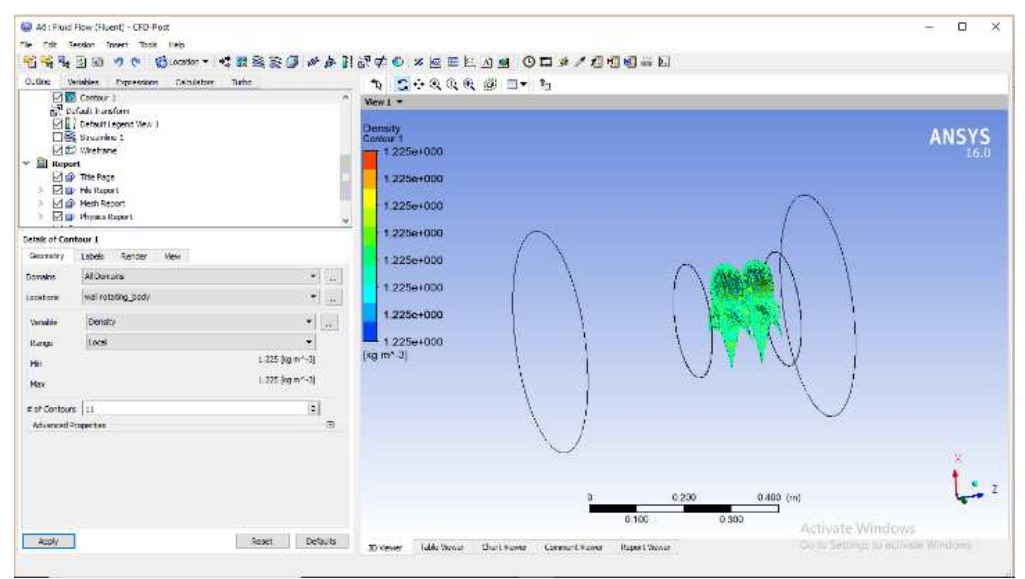

Figure 9: Density of Tandem Propeller 


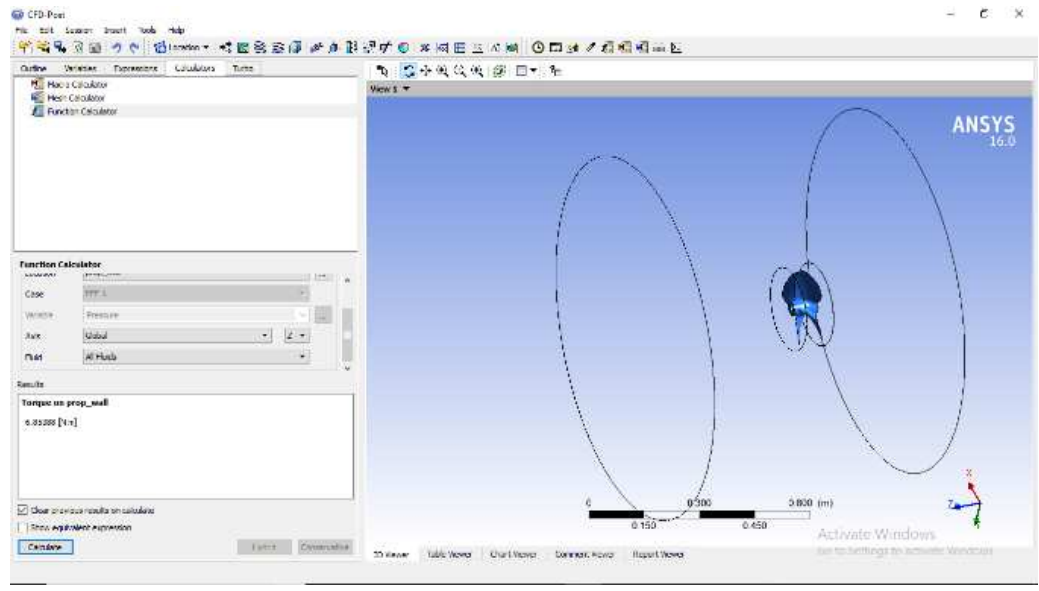

Figure 10: Torque of Single Propeller

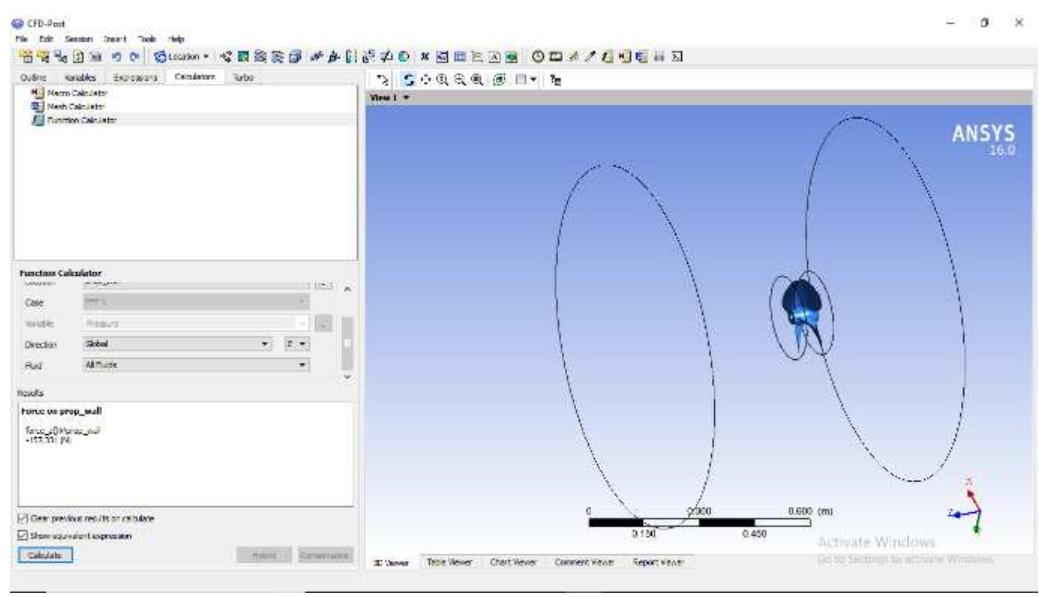

Figure 11: Force of Single Propeller

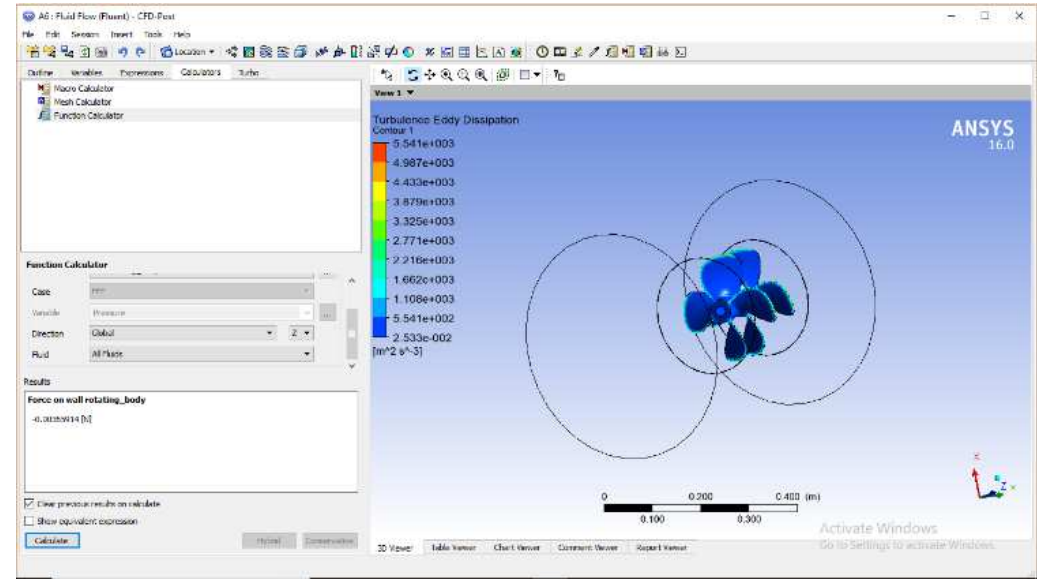

Figure 12: Force of Tandem Propeller 


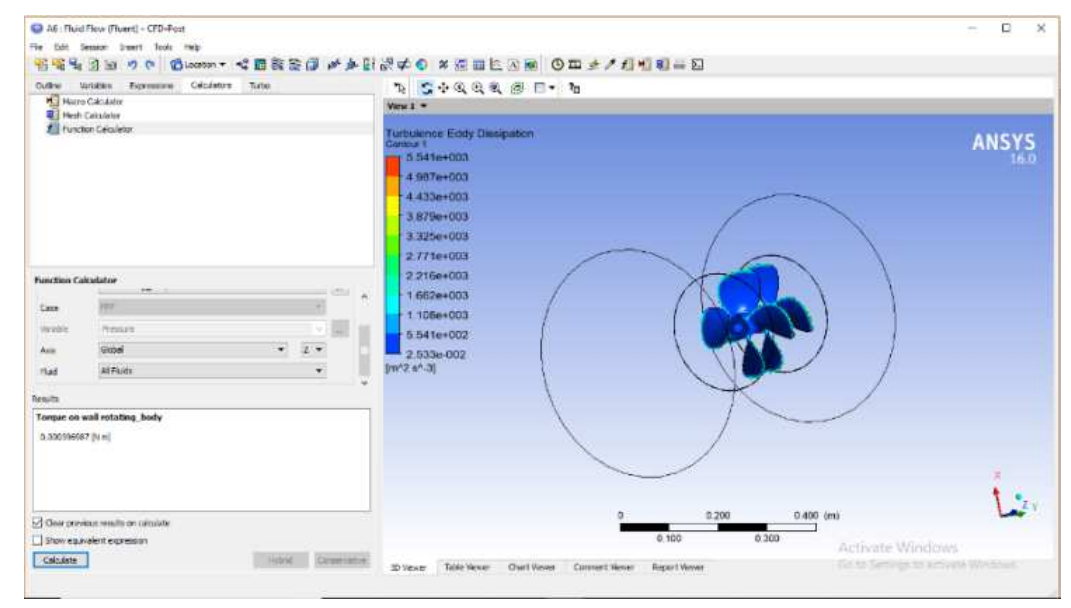

Figure 13: Torque of Tandem Propeller

Force and torque of the single propeller are less when compared to the force and torque of the tandem propeller thereby improving the efficiency of the tandem propeller when compared with the single propeller. This shows us that the tandem propeller gives more thrust and efficiency than that of the single propeller when compared at the same speeds and rpms.

\section{VALIDATIONS OF RESULTS}

Computational validation of the hydrodynamic parameters of the fore propeller in the tandem propeller and tandem propeller in non-cavitating flow, for $\mathrm{J}=0.71$.

Table 3: Single Propeller

\begin{tabular}{|c|c|c|c|}
\hline Parameters & Paper Value & Obtained Value & Error \% \\
\hline $\mathrm{K}_{\mathrm{t}}$ & 0.240 & 0.261 & $8 \%$ \\
\hline $10 \mathrm{~K}_{\mathrm{q}}$ & 0.436 & 0.490 & $10 \%$ \\
\hline$\eta_{0}$ & 0.476 & 0.528 & $10 \%$ \\
\hline
\end{tabular}

Table 4: Tandem Propeller

\begin{tabular}{|c|c|c|c|}
\hline Parameters & Paper Value & Obtained Value & Error \% \\
\hline $\mathrm{K}_{\mathrm{t}}$ & 0.383 & 0.356 & $7 \%$ \\
\hline $10 \mathrm{~K}_{\mathrm{q}}$ & 0.774 & 0.843 & $9 \%$ \\
\hline$\eta_{0}$ & 0.557 & 0.501 & $10 \%$ \\
\hline
\end{tabular}

In the results here we can clearly see that $\mathbf{K}_{\mathbf{t}}$ has the least error when compared to the paper simulations. Thus, it is being considered as the primary value for a baseline.

Other values also have an error of less than $10 \%$, making the simulation carried out to be in close approximation to the original experiment.

\section{CONCLUSIONS}

In this paper, numerical analysis was executed to observe the standard cavitation of the INSEAN E779A propeller in simple and torque propellers. The numerical simulation was actually produced for INSEAN Propeller E779A in currents without cavitation and calculated the coefficients of force and torque. Although, the model disappoints to adequately repeat all the different types of cavitation that may appear. From the previous simulation, we can show that the tandem helix 
provides more thrust and torsion when compared to a single helix in the same flow conditions, which causes a drastic reduction in the cavitation produced.

For the torque propeller, the results show that the conduction of the helices in the non-cavitation current is subjectively anticipated. Regardless of the marginal reduction in the effective pair in case of cavitation, the pair's simultaneous spin propeller affirms its utility in high stacking conditions.

\section{REFERENCES}

1. D. Boucetta, O. Imine, "Numerical Simulation of the cavitating flow around marine co-rotating tandem propellers," Aeronautics and propulsive systems laboratory, Department of marine engineering, 2017

2. Anna Maria Kozlowska, Sverre Steen, "Experimental analysis on the risk of vortex ventilation and the free surface ventilation of marine propellers," Applied Ocean Research, vol.67, pp. 201-212, 2012

3. Qin Wu, Biao Huang, Guoyu Wang, Shuliang Cao, Mingming Zhu, "Numerical modeling of unsteady cavitation and induced noise around a marine propeller," Ocean Engineering, vol.160, pp.143-155, 2018.

4. M.M. Gaafary, H.S. El-Kilani, M.M. Moustafa, “Optimum design of B-series marine propellers,” Alexandria Engineering Journal, vol. 50, pp. 13-18,2011

5. Yu-chi Chang, Chin-ning Hu, Jing-chin Tu, Yi-Chih Chow, "Experimental investigation and numerical prediction of cavitation incurred on propeller surfaces,” Journal of hydrodynamics, vol.22, pp.764-769, 201.

6. BatuhanAktas, Mehmet Atlar, Patrick Fitzsimmons, Weichao Shi, “An advanced joint time-frequency analysis procedure to study cavitation induced noise by using standard series propeller data," Ocean Engineering, vol.170, pp. 329-350, 2018

7. EminKorkut, MehmetAtlar, "An experimental investigation of the effect of foul release coating application on performance, noise and cavitation characteristics of marine propellers, " Ocean Engineering, vol. 41, pp. 1-12, 2012

8. Yu-chi Chang, Chin-ning Hu, Jing-chin Tu, Yi-chin Chow, "Experimental investigation and numerical prediction of cavitation incurred on propeller surfaces, "Journal of Hydrodynamics, vol.22, pp.764-769, 2010

9. YiannisVentikos, George Tzabiras, "A numerical method for the simulation of steady and unsteady cavitating flows," Computers \& Fluids, vol. 29, pp.63-88, 2000

10. Garg, B., \& Agrawal, A. Upswing the Bandwidth and Diminish the Size of Rectangular Flat Panel Directional Device with Metamaterial Cover

11. Lianzhou Wang, ChunyuGuo*, Yumin Su, Pei Xu, Tiecheng Wu, "Numerical analysis of a propeller during heave motion in cavitating flow," Applied Ocean Research, vol.66, pp. 131-145, 2017 
